\title{
Mass Customized Large Scale Production System with Learning Curve Consideration
}

\author{
KuoWei Chen and Richard Lee Storch \\ Industrial \& Systems Engineering, University of Washington, Seattle, U.S.A \\ $\{$ kwc206, rlstorch\}@uw.edu
}

\begin{abstract}
This paper presents the structure for modeling a large scale production system with learning curve considerations. The model will be used to develop a scheduling method that facilitates the production performance for mass customized products. Mass customization is an important manufacturing management strategy but it might lead to unnecessary production losses. Most manufacturing systems' throughput is constrained by one or more bottlenecks and the critical bottleneck may shift from one work station to another. The proposed scheduling method will consider learning curve effects and employ the concept of Shifting Bottleneck Procedure to guide production scheduling decisions. Ultimately, the goal is to improve the throughput of large scale assembly manufacturing systems. A simulation model of a wind turbine assembly line case study is also presented to validate the capability of the proposed method.
\end{abstract}

Keywords: Mass customization, large scale production, learning curves, shifting bottleneck procedure, simulation.

\section{$1 \quad$ Introduction}

This paper focuses on large scale product industries which are known to produce or assemble large scale products. Examples are commercial airplanes, ships, and wind turbines. A large scale production system often consists of a group of structured organizations to produce a family of complex and customized products in a certain geographic region [1]. There are several crucial characteristics among these industries and the nature of a large scale production system is influenced by its products. The most common characteristics of a large scale production system are long cycle time, low total throughput and limited buffer space. For instance, the total assembly cycle time of a wind turbine nacelle may be up to sixteen hours to be completed and the throughput is limited to five units per week. The buffer space or inventory space may be limited. Long cycle time leads to the limited total production volume in the large scale product manufacturing industry which means total throughput, the average output of a production process per unit time, is limited. In order to improve the total throughput, manufacturing engineers are striving to develop more efficient production process methods.

Mass customization (MC) has been a well known contemporary manufacturing industry management technique, enabling a company to compete with their competitors 
in the diverse market. Broadly, the concept of mass customization was first introduced by Davis [2] and he described that manufacturing companies tended to produce goods to serve their relatively large market; meanwhile, satisfying specific needs of individual consumers by customizing products with acceptable cost. However, it is not always free to implement product customization in a production line and the common impact of mass customization on manufacturing trade-offs are important system performances: cost, process time, quality, delivery and volume [3]. Such challenges need to be overcome by those companies who are struggling to achieve mass customization and must consider those performance factors. In spite of a large number of practical approaches and processes for customized product discussed in the literature [4-6], a link between product customization and bottleneck based heuristic with learning curve consideration is still a research area that needs investigation.

This paper proposes the use of learning curve consideration in a bottleneck selection scheduling heuristic to improve the total completion time or system throughput under mass customized large scale product industry. Manufacturers can improve the total throughput by decreasing process time in the bottleneck stage. Those customized products with the similar process on a certain machine are defined as having learning effect when those products are scheduled consecutively. As a result, the customized production system will be closer to the mass production system and then improve the total completion time and throughput. We give a description of bottleneck based heuristic and learning curve effect in Section 2. Section 3 demonstrates the use of learning curve in a bottleneck based scheduling heuristic. Section 4 provides a simulation example which applied the method under a simulated large scale production industry. Section 5 presents the conclusion and future work.

\section{Background}

\subsection{Bottleneck Based Heuristic}

Manufacturing involves many operations on many machines and effective management requires many decisions. There have been many classical scheduling theories for the deterministic problem of scheduling a fixed number of jobs on a given set of machines to improve the production performance such as minimizing makespan, minimizing tardiness, and minimizing lateness. The classical job shop problem can be described as a set of machines that process operations on jobs. Each job has a predefined operation schedule on each machine with the required process time on it [7]. These algorithms can be classified as optimization and approximation algorithms [8, 9]. Among those approximation algorithms, Adams et al. [10] proposed the Shifting Bottleneck (SB) procedure and it is probably the most well known among all heuristics for job shop scheduling problem. A brief statement of the Shifting Bottleneck procedure is as optimizing the sequence on each machine repeatedly, while keeping the sequence of other machines fixed. Each one machine solution is compared with the all others and the machine is identified as the bottleneck machine based on their solution. The SB procedure terminates once all machines have been scheduled. It is generally characterized by following steps to minimize the makespan: bottleneck identification, bottleneck selection, and sequence re-optimization. 


\subsection{Learning Curve Effect}

Learning effect has received considerable attention in management science and it can be described as a steady decline in process time when operators perform the same task repeatedly. Dr. Wright [11] first introduced his famous $\log 2$ learning curve model in 1936 and this model is still one of the best ways to predict operator's performance. Biskup [12,13] might be the pioneer to consider the connection between learning effect and scheduling problem and proved that single-machine problem with the consideration of leaning effects remains polynomially solvable for two objectives which are the minimized deviation from a common due date and minimized sum of flow time. Then he introduced a modified formulation to describe the learning effects in scheduling problem with $p_{i}, i=1, \ldots, n$ being the normal process time and $p_{i r}$ as the process time of job $i$ if it is scheduled in position $r$ in a sequence (1) as below:

$$
p_{i r}=p_{i} r^{a}
$$

where $a \leq 0$ is the learning index, given as the logarithm to the base 2 of the learning rate, and thus for the $80 \%$ hypothesis $a=\log _{2} 0.8=-0.322$ holds. According to the equation above, the needed process time in position $r$ decreases by the number of repetitions which represents that operators gain experience and skills after repetitive operations.

Lee et al. [14] discussed the goal of minimizing total completion time for the two machine flow shop problem. They assumed that the same job has different learning effect on different machines and this assumption is common for those multi-machine scheduling problems with learning consideration. A modified equation (2) with learning effect was introduced by Lee and Wu to represent $p_{i j r}$ as the process time of job $i$ if it is scheduled in position $r$ in a sequence on the machine $j$ :

$$
p_{i j r}=p_{i j} r^{a}
$$

where $j=1, \ldots, m$ is the machine index. With respect to the equation above, the needed process time in position $r$ on machine $j$ decreases by the number of repetitions, hence, different processes on each machine might affect the learning situation. According to results in [13-15] scheduling problem with learning curve consideration is considered to have practical impact to manufacturing schedule decision. Besides, it has shown the capability to improve the mass customization production system.

\section{Methodology}

The method we propose is based on the concept of Shifting Bottleneck procedure [10] with learning curve consideration in a large scale customized product production environment. The objective is to improve production performance such as total completion time or throughput by assigning products which have similar process on the bottleneck stage consecutively in order to reduce the process time by utilizing the 
characteristic of learning curve. Jobs are subject to the constraints that (i) the sequence of machines for each job is prescribed; and (ii) each machine can process only one job at a time. Besides, the processing time of each job might be effected by learning effect when similar processes are scheduled on the same machine consecutively and this causes the processing time of each job on each machine to be changed. Figure 1 presents a flow chart of schedule decision by using bottleneck selection with learning effect consideration.

We start by defining system variables. For instance, $i$ denotes the serial number of job, $j$ denotes the machine or working stage, and $k$ denotes the customization category. Steps 1 and 2 are key steps in this procedure to determine which work station is the real system bottleneck by different criteria. We start to check whether there is any current processing job in the system. If not, the first job is allowed to access into the system according to different dispatching rules and we use first come first served rule here. Otherwise, each work station needs to be measured and analyzed in terms of bottleneck detection criteria. There are basically two well known methods to define the bottleneck in a manufacturing system. The first method is to measure utilization of each work station and the one with highest utilization is considered as a bottleneck. However, it might not be sensitive enough to detect the real bottleneck while there are more than one work stations with same utilization. The second method defines bottleneck by measuring the longest queue line of unprocessed jobs in front of each work station or longest waiting time of each work stage. It is also a simple method to observe the length of queue on each machine in a manufacturing production line. The drawback of this method is lack of accuracy when the maximum of buffer size is limited. The number of unprocessed jobs on more than one busy work stations is restricted by buffer size and this causes difficulty to define a real bottleneck. This is a common situation in the real life especially for large scale production system, for example, an airplane assembly line may just have one buffer space in front each stage. Therefore, we choose the largest waiting time as a bottleneck selection criterion in this paper:

$$
\max \left(\frac{\sum_{i=1}^{n} t_{i 1}-c_{i 0}}{i}, \frac{\sum_{i=1}^{n} t_{i 2}-c_{i 1}}{i}, \ldots, \frac{\sum_{i=1}^{n} t_{i j}-c_{i j-1}}{i}\right)
$$

for all $j$,where $j=1 \ldots m$ being machine index, $t_{i j}$ denotes the arrival time of job $i$ on machine $j$ and $c_{i j}$ is the completion time of job $i$ on machine $j$.

Once the bottleneck station is determined, we go to the next step to identify relevant job information in order to make schedule decision. Steps 3 and 4 should be performed at anytime to those unprocessed jobs in front of the main system. Under the customization production environment, the information of each job is assumed to be well prescribed according to customer's requirement; however, the assembly 


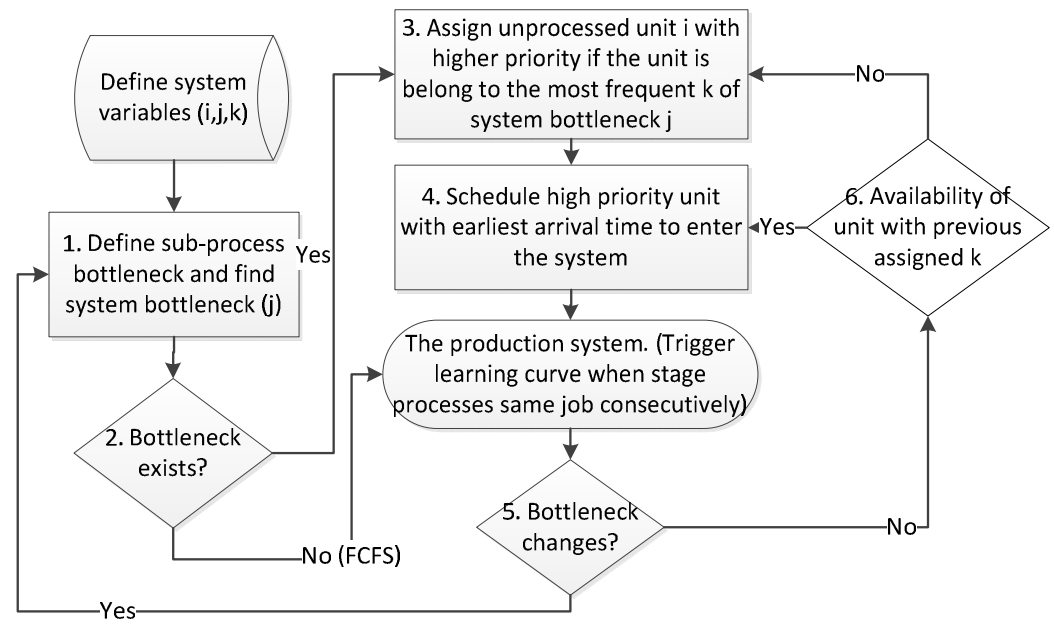

Fig. 1. Steps of proposed scheduling procedure with learning effect

sequence can be assigned by the manufacturer to achieve flexible manufacturing. Next, this model accumulates the number for each option in the corresponding work station. Once the bottleneck is determined in Steps 1 and 2, this model assigns those jobs which are in the same job family with most frequent number in the queue of unprocessed jobs corresponding to bottleneck stage with higher priority. The purpose of this step is to accumulate the same process on the bottleneck stage and then learning effect can be triggered to reduce the process time. This step always schedules those jobs with higher priority first to enter the main system unless the bottleneck changes or there are no more jobs defined as higher priority in the queue of unprocessed jobs in front of the main system. We go back to steps 1 and 2 to redetermine the new bottleneck immediately when the bottleneck changes. Otherwise, this model keeps on steps 3 and 4 to search for the most frequent job family and assign them with higher priority to access into the system if those jobs in the previous majority job family have been all processed to enter the main system.

The final step implements the learning curve on the bottleneck machine to reduce process time when a job is still in the same job family as the previous one. There are numerous descriptions and practical applications of learning curve discussed in the literature $[13,16]$, however, the proposed heuristic in this paper uses the classic Wright's $\log 2$ learning curve model given a learning index $a=\log _{2} 0.8=-0.322$ for the $80 \%$ hypothesis.

\section{Simulation and Results}

The proposed procedure in section 3 is applied in a simulated example for a wind turbine assembly industry and this model is carried out to analyze the simulation results using the ARENA simulation software. The example assumes a wind turbine company can offer its customers customized nacelles with 3 options $(k=1 \ldots 3)$ on 15 
different categories ( $j=1, \ldots, 15)$ without supply shortage or other unexpected emergent situation such as labor absence or delay of delivery and the simplified process flow is presented in Figure 2. On the other hand, these jobs all need to process through 15 work stations to be completed with same process sequence in this complex system. There are a total of 700 unprocessed jobs with continuous arrival time and the information of each job is prescribed. This example assumes the buffer space of each work station is restricted to 2 as well as the bottleneck is determined by measuring the largest waiting time.

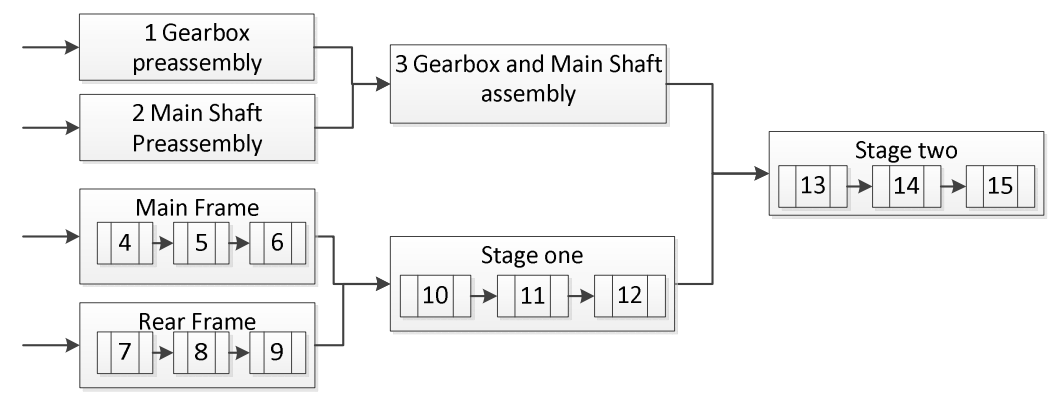

Fig. 2. Simplified process flow of nacelles assembly line

According to figure 3, the dash trend presents a schedule decision with traditional first come first serve dispatching rule (FCFS) which means a job is scheduled according to their arrival time. The earlier a job arrives in the queue in front of the main system, the earlier it can be processed into the main system. The solid trend presents a schedule decision using proposed scheduling procedure with learning curve consideration (LC) and it shows that the average completion time has been improved and reduced from around 4016 minutes to 3450 minutes in this wind turbine example.

By following the proposed procedure, every job that arrives in the queue in front of the main system must be checked by the model to determine if it meets the criteria as a higher priority level. It seems that the stage 2 is defined as the primary bottleneck and it consists of sub-processes 13, 14, and 15. Figure 4 (a) (b) (c) depict the process time of individual jobs on each work station respectively. It can be easily seen that learning curve happens on all of three work stations, especially on station (13) and station (14). This also indicates that a work station is determined as a critical bottleneck when there is an exponential decrease period influenced by learning effect. However, a bottleneck might go along with a short learning effect period and this just means the bottleneck changes to another work station quickly in a short time. Besides, learning effect may happen on a non-bottleneck work station according to random combinatorial sequence and this non-bottleneck work station can also benefit by the nature of learning effect. 


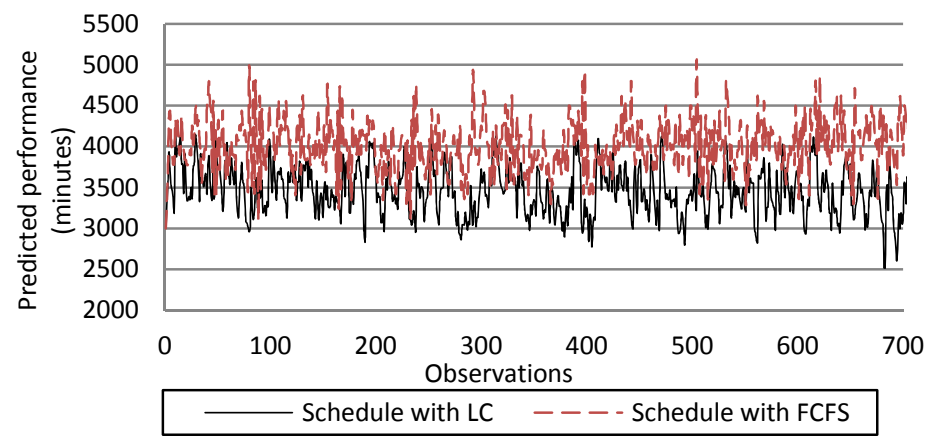

Fig. 3. A comparison of predicted completion time

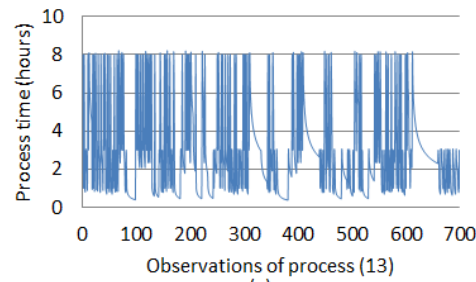

(a)

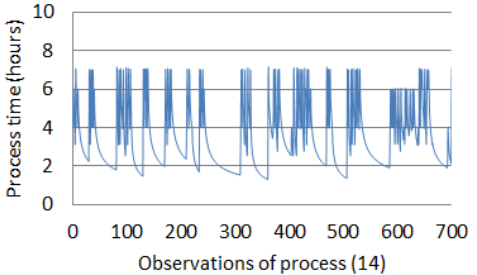

(b)

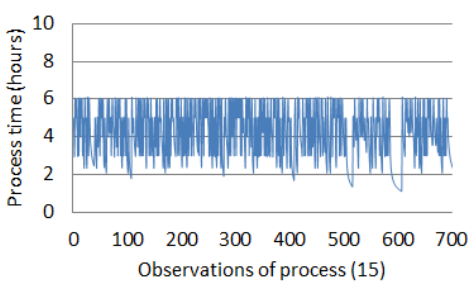

(c)

Fig. 4. Process time of each job in the bottleneck stage

\section{$5 \quad$ Conclusions and Future Work}

In the present paper, we propose a structure of modeling with learning effect under mass customization production system. A simulation model also is developed to demonstrate this procedure and analyze the results according to a simulated wind turbine assembly line example. The total completion time has a significant improvement by using the proposed scheduling procedure. This paper concluded that this heuristic has potential capability to improve the performance of a customized production system by assigning job family on bottleneck stage to reduce the process time. In addition, it also can provide manufacturers better scheduling decision support to satisfy customer's demand.

Future development of the method may include (a) using different bottleneck detection method to determine a real bottleneck in a more accurate and sensitive way 
(b) comparing the result by different learning curve models, and (c) analyzing the influence of other objectives to the proposed heuristic such as minimizing tardiness or minimizing the variation of completion time. We selected the total completion time as the objective in this paper. Additionally, more complicated and therefore more realistic production systems will be considered.

\section{References}

1. Lu, R.F., Petersen, T.D., Storch, R.L.: Modeling customized product configuration in large assembly manufacturing with supply-chain considerations. International Journal of Flexible Manufacturing Systems 19(4), 685-712 (2008)

2. Davis, S.M.: From "future perfect": Mass customizing. Strategy \& Leadership 17(2), 16-21 (1989)

3. Squire, B., et al.: The impact of mass customisation on manufacturing trade-offs. Production and Operations Management 15, 10-21 (2006)

4. Silveira, G.D., Borenstein, D., Fogliatto, F.S.: Mass customization literature review and research directions. International Journal of Production Economics 72, 1-13 (2001)

5. Ulrich, K.T., Eppinger, S.D.: Product Design and Development, 2nd edn. McGraw-Hill (2000)

6. Huang, X., Krista, M.M., Schroeder, R.G.: Linking learning and effective process implementation to mass customization capability. Journal of Operations Management 26(6), 714-729 (2008)

7. Blazewicz, J., Domschke, W., Pesch, E.: The job shop scheduling problem Conventional and new solution techniques. European Journal of Operational Research 93, 1-33 (1996)

8. Jain, A.S., Meeran, S.: Deterministic job-shop scheduling Past, present and future. European Journal of Operational Research 113, 390-434 (1999)

9. Wenqi, H., Alihua, Y.: An improved shifting bottleneck procedure for the job shop scheduling problem. Computers \& Operations Research 31(12), 2093-2110 (2004)

10. Adams, J., Balas, E., Zawack, D.: The Shifting Bottleneck Procedure for Job Shop Scheduling. Management Science 34, 391-401 (1988)

11. Wright, T.P.: Factors Affecting the Cost of Airplanes. Journal of Aeronautical Sciences 3, 122-128 (1936)

12. Biskup, D.: Single-machine scheduling with learning considerations. European Journal of Operational Research 115, 173-178 (1999)

13. Biskup, D.: A state-of-the-art review on scheduling with learning effects. European Journal of Operational Research 188(2), 315-329 (2008)

14. Lee, W.-C., Wu, C.-C.: Minimizing total completion time in a two-machine flowshop with a learning effect. International Journal of Production Economics 88(1), 85-93 (2004)

15. Anzanello, M.J., Fogloatto, F.S.: Learning curve models and application: Literaure review and research directions. International Journal of Industrial Ergonomics 41, 573-583 (2011)

16. Roser, C., Nakano, M., Tanaka, M.: Shifting Bottleneck Detection. In: Simulation Conference, vol. 2, pp. 1079-1086 (2002) 